\title{
Performance of a PET Insert for High-Resolution Small-Animal PET/MRI at 7 Tesla
}

\author{
Greg Stortz ${ }^{1}$, Jonathan D. Thiessen ${ }^{2,3}$, Daryl Bishop ${ }^{4}$, Muhammad Salman Khan ${ }^{5}$, Piotr Kozlowski ${ }^{6}$, Fabrice Retière ${ }^{4}$, \\ Graham Schellenberg ${ }^{7}$, Ehsan Shams ${ }^{8}$, Xuezhu Zhang ${ }^{9,10}$, Christopher J. Thompson ${ }^{11}$, Andrew L. Goertzen*9,7, \\ and Vesna Sossi*1
}

\begin{abstract}
${ }^{I}$ Department of Physics and Astronomy at the University of British Columbia, Vancouver, British Columbia, Canada; ${ }^{2}$ Department of Medical Biophysics, Western University, London, Ontario, Canada; ${ }^{3}$ Graduate Program in Biomedical Engineering, University of Manitoba, Winnipeg, Manitoba, Canada; ${ }^{4}$ Detector Development Group, TRIUMF, Vancouver, British Columbia, Canada; ${ }^{5}$ Department of Electrical Computer Engineering, University of Manitoba, Winnipeg, Manitoba, Canada; ${ }^{6}$ Department of Radiology, University of British Columbia, Vancouver, British Columbia, Canada; ${ }^{7}$ Department of Physics and Astronomy, University of Manitoba, Winnipeg, Manitoba, Canada; ${ }^{8}$ Biomedical Engineering Graduate Program, University of Manitoba, Winnipeg, Manitoba, Canada; ${ }^{9}$ Department of Radiology, University of Manitoba, Winnipeg, Manitoba, Canada; ${ }^{10}$ Department of Biomedical Engineering, University of California, Davis, Davis, California; and ${ }^{11}$ Montreal Neurological Institute, McGill University, Montreal, Quebec, Canada
\end{abstract}

\begin{abstract}
We characterize a compact MR-compatible PET insert for simultaneous preclinical PET/MRI. Although specifically designed with the strict size constraint to fit inside the 114-mm inner diameter of the BGA-12S gradient coil used in the BioSpec 70/20 and 94/20 series of small-animal MRI systems, the insert can easily be installed in any appropriate MRI scanner or used as a stand-alone PET system. Methods: The insert consists of a ring of 16 detector-blocks each made from depth-of-interaction-capable dual-layer-offset arrays of cerium-doped lutetium-yttrium oxyorthosilicate crystals read out by silicon photomultiplier arrays. Scintillator crystal arrays are made from $22 \times 10$ and $21 \times 9$ crystals in the bottom and top layers, respectively, with respective layer thicknesses of 6 and $4 \mathrm{~mm}$, arranged with a 1.27- $\mathrm{mm}$ pitch, resulting in a useable field of view $28 \mathrm{~mm}$ long and about $55 \mathrm{~mm}$ wide. Results: Spatial resolution ranged from 1.17 to $1.86 \mathrm{~mm}$ full width at half maximum in the radial direction from a radial offset of $0-15 \mathrm{~mm}$. With a $300-$ to $800-\mathrm{keV}$ energy window, peak sensitivity was $2.2 \%$ and noise-equivalent count rate from a mouse-sized phantom at $3.7 \mathrm{MBq}$ was $11.1 \mathrm{kcps}$ and peaked at $20.8 \mathrm{kcps}$ at $14.5 \mathrm{MBq}$. Phantom imaging showed that features as small as $0.7 \mathrm{~mm}$ could be resolved. ${ }^{18} \mathrm{~F}-\mathrm{FDG}$ PET/MR images of mouse and rat brains showed no signs of intermodality interference and could excellently resolve substructures within the brain. Conclusion: Because of excellent spatial resolvability and lack of intermodality interference, this PET insert will serve as a useful tool for preclinical PET/MR.
\end{abstract}

Key Words: animal imaging; image reconstruction; instrumentation; molecular imaging; PET/MRI; SiPM

J Nucl Med 2018; 59:536-542

DOI: 10.2967/jnumed.116.187666

Received May 1, 2017; revision accepted Sep. 11, 2017.

For correspondence or reprints contact: Greg Stortz, University of British Columbia, Hennings 325, 6224 Agricultural Rd., Vancouver, BC V6T 1Z1, Canada.

E-mail: greg.stortz@alumni.ubc.ca

${ }^{*}$ Contributed equally to this work.

Published online Sep. 14, 2017.

COPYRIGHT (C 2018 by the Society of Nuclear Medicine and Molecular Imaging.
$\mathbf{P}$ ET allows for functional in vivo imaging of a positron-emitting radiotracer. While the strength of PET lies in the ability to accurately detect radioisotopes at picomolar concentrations in vivo (1), PET does not provide anatomic information. Hybrid PET systems incorporating CT or MRI dramatically increase the value of PET by providing an anatomic backdrop to PET images.

Although PET/CT is a mature clinical and preclinical technology, PET/MR is still an emerging technology. PET/MR has traditionally been a challenge because the photomultiplier tubes that detect scintillation light cannot function inside a strong magnetic field. Early attempts at simultaneous PET/MR for clinical (2-4) and preclinical applications $(5,6)$ used fiber-optic cables to direct scintillation light to photomultiplier tubes positioned outside the magnetic field. In addition to signal distortion and energy resolution degradation due to loss of light, the volume of fiber-optic cabling created space constraints limiting the number of detectors.

Later work used MR-compatible avalanche photodiodes to directly read out scintillator crystals inside an MRI scanner (7-10). Compared with photomultiplier tubes, avalanche photodiodes result in poor timing resolution and low, temperature-sensitive gain (11). Silicon photomultipliers (SiPMs) have recently become an attractive alternative to avalanche photodiodes for PET/MR (12-14) because they offer gain and timing resolution comparable to photomultiplier tubes while also functioning in strong magnetic fields with little temperature dependence $(11,15,16)$.

This work presents an SiPM-based MR-compatible high-resolution PET insert allowing for simultaneous PET/MR imaging of small animals. An insert is advantageous to integrated PET/MR systems because it can be retrofit to existing MRI scanners, potentially reducing the capital cost by a large factor for groups pursuing simultaneous PET/MR, while also functioning as a compact standalone PET system. Our design was constrained so that the insert could fit inside a Bruker BGA-12S gradient coil (inner diameter, $114 \mathrm{~mm}$ ) installed in both the 70/20 and the 94/20 models of the Bruker BioSpec preclinical MRI scanner. The inner bore of the PET insert was required to be large enough to accommodate the $60-\mathrm{mm}$ outer diameter of the Bruker 35-mm radiofrequency volume coil. The 


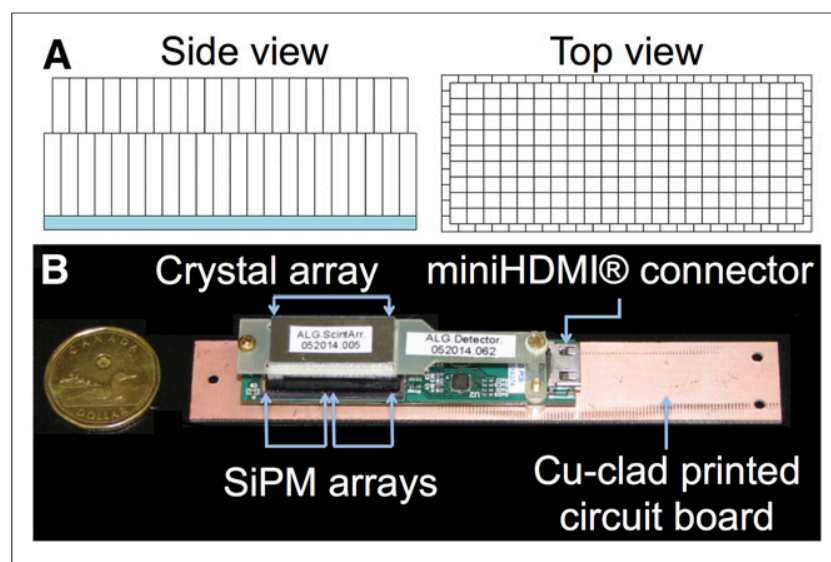

FIGURE 1. Schematic of crystal array, with light-guide shown in blue (A), and photograph of assembled detector-block next to Canadian $\$ 1$ coin for size comparison (B).

resulting high curvature of the PET detector ring exacerbates resolution degradation from the parallax effect, as annihilation photons originating from even a small radial offset will enter the detectors at a substantial angle. Our PET system was therefore built using depth-ofinteraction-capable detectors to mitigate the parallax effect.

We previously reported on the MR compatibility of the PET insert (17) and have shown the first images and a preliminary characterization (18). Here we present a final characterization of the PET insert after major firmware updates that yielded substantial improvements in signal timing accuracy and count rate performance and therefore superior image quality. Additionally, a point-spread-function-modeling maximum-likelihood expectationmaximization (PSF-MLEM) algorithm tailored to this PET detector geometry (19) was used for the first time to reconstruct phantom and rodent data.

\section{MATERIALS AND METHODS}

\section{PET Insert}

Each detector-block consists of a dual-layer-offset array $(20,21)$ of cerium-doped lutetium-yttrium oxyorthosilicate crystals read out by two ArraySB-4 SiPM arrays (SensL Inc.). Crystal arrays (Proteus Inc.) are made from $22 \times 10(1.2 \times 1.2 \times 6 \mathrm{~mm})$ crystals in the bottom layer and $21 \times 9(1.2 \times 1.2 \times 4 \mathrm{~mm})$ crystals in the top layer. Crystals are polished and coated in enhanced specular reflector film bonded with Dymax OP-20 ultraviolet curing glue and arranged with a $1.27-\mathrm{mm}$ pitch as shown in Figure 1A. A 1.0-mm-thick glass light-guide allows scintillation light to diffuse before reaching the SiPMs. The 32 SiPM analog outputs are multiplexed to four by a resistive charge-division network $(22,23)$. Analog outputs are carried from each detector-block by high-definition multimedia interface cables, which also power the SiPMs (24).

Sixteen detector-blocks were mounted to copper-clad printed circuit boards, which are mounted to plastic supports secured to a 60-mm-wide carbon-fiber tube. The geometry of the PET insert is summarized in Table 1. Figure 1B shows an assembled detector-block. Figure 2A shows the partially assembled gantry, making visible the $35-\mu \mathrm{m}$-thick copper foil on the printed circuit boards and lining the inside of the gantry for radiofrequency shielding from the MRI (17). The assembled gantry was sheathed in a woven carbon-fiber tube (Rockwest Composites) and closed with machined plastic caps (Fig. 2B).
Four detector-support boards supply power to the detectors and relay analog signals to detector boards in an OpenPET data acquisition system $(25,26)$ via a 96-pin cable. Triggering of the OpenPET system is based on a voltage threshold, after which digitized (peak minus baseline) time-stamped signals are reported back to an acquisition computer via universal serial bus 2.0. OpenPET firmware was highly customized to output 8-byte singles packets, allowing acquisition of singles at rates of up to 5 Mcps.

\section{Data Processing}

Crystal identification and coincidence detection are performed retrospectively by software. An automated algorithm to segment flood histograms allows for rapid generation of crystal and energy look-up tables (27) used for crystal identification and energy discrimination (with a 300 - to $800-\mathrm{keV}$ window). The coincidence window was set to $10 \mathrm{~ns}$ (appropriate for the measured 5.4-ns singles timing resolution). Randoms rates were estimated using a delayed coincidence window.

Each crystal pair was mapped to a sinogram bin using nearestneighbor interpolation to evenly spaced radial and angular intervals. Because complex geometric factors following from the dual-layeroffset detector design result in a situation where it is not possible to use conventional axial mashing approaches (as described by Michelograms (28)) to create a single set of sinograms, an alternative method was developed (29). In this work, sinograms were formed using 67 radial bins, 104 views, a span of 3, and a maximum ring difference of 19. Reconstructed sinograms were normalized with component-based normalization. Analytic reconstruction was performed using the filtered backprojection (FBP)-3-dimensional reprojection (3DRP) algorithm as implemented in the open-source reconstruction suite STIR (Software for Tomographic Image Reconstruction), version 3.0 (30).

\section{PSF-MLEM Algorithm}

A line-of-response-based PSF-modeling MLEM algorithm developed in-house was previously shown to produce images of very high contrast when tested on simulated data (19), but without incorporation

TABLE 1

Geometry and Features of PET Insert

\begin{tabular}{ll}
\hline \multicolumn{1}{c}{ Parameter } & \multicolumn{1}{c}{ Description } \\
\hline $\begin{array}{l}\text { Detector } \\
\text { Photosensor }\end{array}$ & $\begin{array}{l}2 \times \text { SensL ArraySB-4 } \\
\text { Cerium-doped lutetium-yttrium } \\
\text { oxyorthosilicate }\end{array}$ \\
\hline $\begin{array}{l}\text { Scintillator } \\
\text { Scintillator array } \\
\text { dimensions }\end{array}$ & $22 \times 10$ (bottom) $+21 \times 9$ (top) \\
\hline $\begin{array}{l}\text { Scintillator crystal size } \\
\text { Crystal pitch }\end{array}$ & $1.2 \times 1.2 \times 6 / 4 \mathrm{~mm}^{3}$ (bottom/top) \\
\hline $\begin{array}{l}\text { Light-guide } \\
\text { System geometry }\end{array}$ & $1.27 \mathrm{~mm}$ \\
\hline $\begin{array}{l}\text { Detectors per ring } \\
\text { Detector ring diameter }\end{array}$ & 16 \\
\hline $\begin{array}{l}\text { PET gantry inner } \\
\text { diameter }\end{array}$ & $65.8 \mathrm{~mm}$ of glass \\
\hline $\begin{array}{l}\text { PET gantry outer } \\
\text { diameter }\end{array}$ & $113 \mathrm{~mm}$ \\
\hline $\begin{array}{l}\text { Axial FOV } \\
\text { Transaxial FOV }\end{array}$ & $27.94 \mathrm{~mm}$ \\
\hline
\end{tabular}




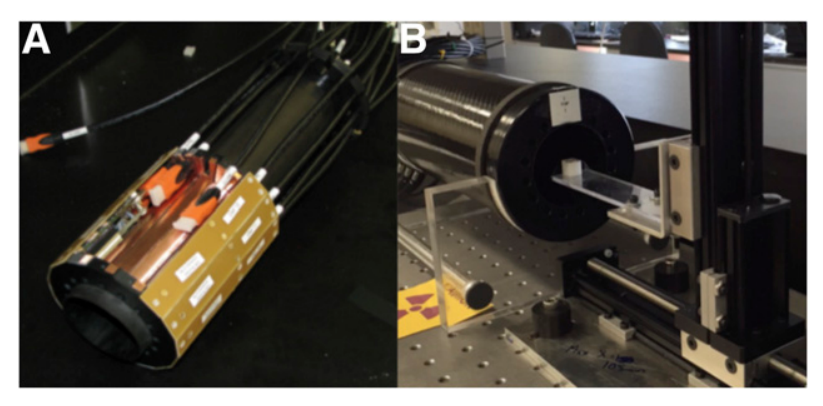

FIGURE 2. Partially assembled ring of PET detector-blocks (A) and assembled PET insert (B).

of normalization. Because the PSF-MLEM system matrix accounts for many of the geometric factors that would be included in componentbased normalization, normalization factors must be different from those for FBP. In an approach similar to that of Bai et al. (31), the system matrix was used to forward-project a known distribution of activity (a thin-walled annulus) so that the geometric factors incorporated in the system matrix could be determined and removed from the normalization factors.

The algorithm was further modified to remove persistent nonuniformity artifacts (28), resulting in a drastic increase in reconstruction time ( 25 iterations taking over $1 \mathrm{y}$ with work split among 20 threads running on a PC with four 12-core AMD Opteron 6192 CPUs). Identification of massive symmetries in the system matrix allowed for less than $1 \%$ of nonzero system matrix elements to be calculated and the rest populated using these symmetries. Instead of using on-the-fly calculation, this small fraction of system matrix elements was computed once and saved to disk (occupying 1.5 or $5.8 \mathrm{~GB}$ of storage space for zoom-1 or -2 reconstructions, respectively) to be recalled during reconstruction, producing a more practical reconstruction time ( 25 or 105 min per iteration for zoom-1 or -2). Although not yet implemented, the ordered-subset expectation-maximization algorithm could further accelerate reconstruction time. For images shown in this paper, iterations were performed until there was no further visual improvement in image contrast.

\section{PET System Performance}

The PET insert was characterized in terms of spatial resolution, sensitivity, and noise-equivalent count rate (NECR), closely following the methods prescribed by the National Electrical Manufacturers Association (NEMA) NU 4-2008 protocol (32). Phantom images along with mouse and rat ${ }^{18} \mathrm{~F}-\mathrm{FDG}$ images further illustrate the performance of the PET insert. Rodent imaging was performed in the 7-T Bruker MRI scanner as MR images were acquired.
Spatial Resolution. A 0.49-MBq 0.25-mm-diameter spherical ${ }^{22} \mathrm{Na}$ source embedded in a 1.0 -cm-wide acrylic cube (model MMS09; Eckert \& Ziegler Isotope Products) was scanned at radial offsets of $0,5,10$, and $15 \mathrm{~mm}$ at the axial center and again offset from the axial center by one quarter the length of the field of view (FOV) with at least $10^{6}$ coincidences acquired per position. Source movement was enabled by a motorized stage with a positioning accuracy of about $15 \mu \mathrm{m}$ over $5 \mathrm{~cm}$ of motion (Fig. 2B; model MN10-150M02-21; Velmex). From reconstructed FBP-3DRP images (voxel size, $0.159 \times 0.159 \times 0.635 \mathrm{~mm})$, the full width at half maximum and full width at tenth maximum were determined according to the protocol defined by the NEMA NU 4-2008 standard. Volumetric resolution (the product of full width at half maximum in the 3 orthogonal directions) was also calculated.

Sensitivity. A ${ }^{22} \mathrm{Na}$ source like the one used to measure resolution but with an activity of $125 \mathrm{kBq}$ was stepped along the axis of the PET scanner with a step size of $0.635 \mathrm{~mm}$ (half the crystal pitch) using the motorized stage, with $30 \mathrm{~s}$ of data acquired at each position. Randoms-corrected sinograms for each source position were formed, and for each view all sinogram bins farther than $1.0 \mathrm{~cm}$ from the highest-count bin were set to zero. The number of counts remaining in all sinograms was summed and divided by the acquisition time to yield the count rate, $C$. From this, sensitivity was calculated as

$$
\text { Sensitivity }=\frac{C}{\gamma A}
$$

Eq. 1

where $\gamma$ is the branching ratio of ${ }^{22} \mathrm{Na}$ for positron decay, and $A$ is the source activity. Sensitivity is plotted as a function of source position along the scanner axis.

NECR and Scatter Fraction. NECR was measured according to the NEMA NU 4-2008 protocol using the mouse-sized count-rate phantom. Beginning with approximately $40 \mathrm{MBq}$ of ${ }^{18} \mathrm{~F}$, data were collected using 10-min frames once per hour for $14 \mathrm{~h}$. Sinograms of prompt and delayed coincidences were created; from these, rates of true $(T)$, random $(R)$, and scattered $(S)$ coincidences were determined, and NECR was calculated according to

$$
\mathrm{NECR}=\frac{T^{2}}{T+S+2 R}
$$

Scatter fraction $[S /(T+S)]$ is reported for a source activity of 3.2 MBq.

Phantom Images. To demonstrate the image quality achievable with this PET system, two phantoms were imaged: the NEMA NU 4-2008 image-quality phantom and a microresolution phantom (model 850.500;

TABLE 2

\begin{tabular}{|c|c|c|c|c|c|c|c|}
\hline \multirow[b]{2}{*}{ Offset (mm) } & \multicolumn{3}{|c|}{ FWHM (mm) } & \multicolumn{3}{|c|}{ FWTM (mm) } & \multirow[b]{2}{*}{ Volume $(\mu \mathrm{L})$} \\
\hline & Radial & Tangential & Axial & Radial & Tangential & Axial & \\
\hline 0 & 1.17 & 1.35 & 1.36 & 2.43 & 3.23 & 2.70 & 2.15 \\
\hline 5 & 1.27 & 1.45 & 1.30 & 2.62 & 3.27 & 2.67 & 2.38 \\
\hline 10 & 1.53 & 1.49 & 1.38 & 3.36 & 3.31 & 2.82 & 3.13 \\
\hline 15 & 1.86 & 1.46 & 1.49 & 4.16 & 3.40 & 3.04 & 4.06 \\
\hline
\end{tabular}

Spatial Resolution at Axial Center of FOV

FWHM $=$ full width at half maximum; FWTM $=$ full width at tenth maximum. 
TABLE 3

Spatial Resolution at One-Quarter Axial Offset from Center of FOV

\begin{tabular}{|c|c|c|c|c|c|c|c|}
\hline \multirow[b]{2}{*}{ Offset $(\mathrm{mm})$} & \multicolumn{3}{|c|}{ FWHM (mm) } & \multicolumn{3}{|c|}{ FWTM (mm) } & \multirow[b]{2}{*}{ Volume $(\mu \mathrm{L})$} \\
\hline & Radial & Tangential & Axial & Radial & Tangential & Axial & \\
\hline 0 & 1.23 & 1.23 & 1.41 & 2.61 & 2.80 & 2.78 & 2.15 \\
\hline 5 & 1.27 & 1.30 & 1.37 & 2.68 & 2.92 & 2.84 & 2.27 \\
\hline 10 & 1.56 & 1.45 & 1.46 & 3.35 & 3.10 & 2.97 & 3.30 \\
\hline 15 & 1.87 & 1.50 & 1.53 & 4.22 & 3.30 & 3.19 & 4.28 \\
\hline
\end{tabular}

FWHM $=$ full width at half maximum; FWTM $=$ full width at tenth maximum .

MI Labs). The 58-mm-long image-quality phantom was filled with 8.6 $\mathrm{MBq}$ of ${ }^{18} \mathrm{~F}$ and scanned in a 3-step step-and-shoot acquisition for 20 min per bed position, enabled by the motorized stage. This stepand-shoot protocol was repeated 4 times, and datasets for the same bed position were appended together. We chose to scan at a higher activity and for a longer time than prescribed by NEMA NU 4-2008 to avoid a dataset with a very low count due to low sensitivity and the need to scan in 3 bed-positions. The microresolution phantom, which has an axial length of $12 \mathrm{~mm}$, was scanned in one 60-min acquisition after being filled with $8.7 \mathrm{MBq}$ of ${ }^{18} \mathrm{~F}$. The phantom contains groups of rods with diameters of $0.7,0.8,0.9,1.0,1.2$, and $1.5 \mathrm{~mm}$. Center-to-center spacing is twice the rod diameter.

Data were reconstructed with FBP-3DRP and the aforementioned PSF-MLEM method. The FBP reconstruction of the NEMA imagequality phantom was analyzed according to the NEMA NU 4-2008 protocol to quantify uniformity, recovery coefficients (mean image value along the center of the rods divided by mean image value in the uniformity region), and spillover ratio (mean value in each of the cold inserts divided by mean value in the uniformity region).

Rodent Images. Simultaneous PET/MR images of both a mouse and a rat were acquired and are presented in this paper to demonstrate the intended use of the PET system. The PET system was centered inside the MRI gradient coil.

A $4.47-\mathrm{MBq}$ dose of ${ }^{18} \mathrm{~F}-\mathrm{FDG}$ was administered to a 40.1 -g 3 xTG mouse by intraperitoneal injection (preferred to intravenous injection because of a higher success rate). After a 1-h uptake period, acquisition of PET and MRI data commenced with the mouse brain centered in the FOV with an $18 \times 22 \mathrm{~mm}$ flat quadrature surface coil

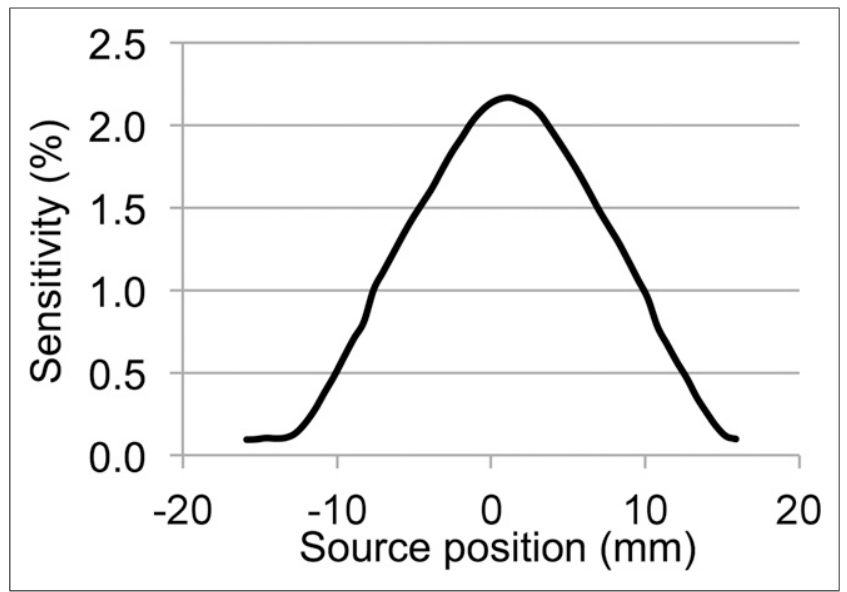

FIGURE 3. Axial sensitivity profile. positioned above the brain. Half-Fourier single-shot turbo spin-echo MRI acquisitions ran for the entirety of the 20-min PET acquisition. The resulting $128 \times 128$ MR images had an in-plane resolution of $0.15875 \mathrm{~mm}$.

A $14.8-\mathrm{MBq}$ dose of ${ }^{18} \mathrm{~F}-\mathrm{FDG}$ was administered to a $270-\mathrm{g}$ Sprague-Dawley rat by intravenous injection. A custom-built radiolucent radiofrequency volume coil (33) was placed inside the PET system, with the rat's brain centered in the FOV. Simultaneous PET/ MRI began after a 50-min uptake period. Half-Fourier single-shot turbo spin-echo acquisitions were run for the entirety of the 30-min PET acquisition, resulting in $128 \times 128$ images with an in-plane resolution of $0.3175 \mathrm{~mm}$.

\section{RESULTS}

The reconstructed spatial resolution throughout the FOV is summarized in Tables 1-3. In the axial center of the FOV, radial full width at half maximum ranged from 1.17 to $1.86 \mathrm{~mm}$ between the radial center and a $15-\mathrm{mm}$ offset, and volumetric resolution ranged from 2.15 to $4.06 \mu \mathrm{L}$.

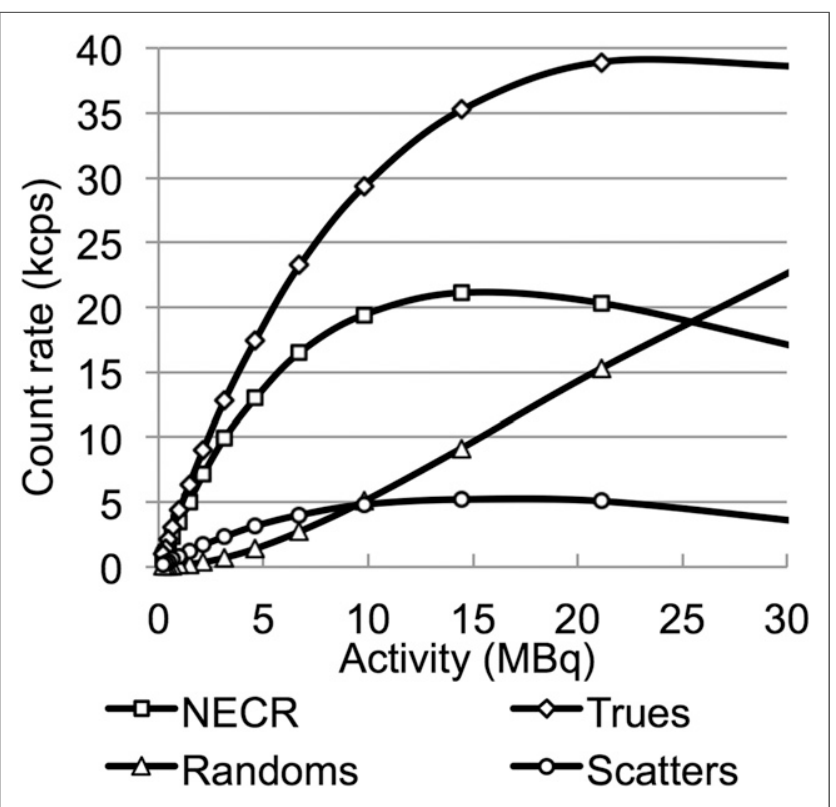

FIGURE 4. NECR as function of source activity for NEMA mousesized phantom, with corresponding rates of true, random, and scatter coincidences. 
TABLE 4

Analysis of NEMA Image Quality Phantom

\begin{tabular}{|c|c|c|c|c|c|c|c|c|}
\hline \multirow[b]{2}{*}{ Parameter } & \multicolumn{3}{|c|}{ Uniformity } & \multicolumn{3}{|c|}{ Recovery coefficient } & \multicolumn{2}{|c|}{ Spillover ratio } \\
\hline & FBP & PSF & $\mathrm{mm}$ & FBP & PSF & Air/water & FBP & PSF \\
\hline Mean & 1.0 & 1.0 & 1 & $0.18 \pm 0.02$ & $0.23 \pm 0.03$ & Air & $0.13 \pm 0.07$ & $0.14 \pm 0.02$ \\
\hline Maximum & 1.3 & 1.2 & 2 & $0.43 \pm 0.03$ & $0.62 \pm 0.06$ & Water & $0.25 \pm 0.06$ & $0.24 \pm 0.03$ \\
\hline Minimum & 0.60 & 0.8 & 3 & $0.64 \pm 0.03$ & $0.78 \pm 0.07$ & & & \\
\hline \multirow[t]{2}{*}{ SD } & 0.076 & 0.06 & 4 & $0.77 \pm 0.02$ & $0.80 \pm 0.05$ & & & \\
\hline & & & 5 & $0.84 \pm 0.03$ & $0.83 \pm 0.07$ & & & \\
\hline
\end{tabular}

The sensitivity profile followed a characteristic triangular shape and peaked at $2.2 \%$ (Fig. 3). Firmware updates led to an improvement in the previously observed sensitivity of $1.9 \%$. The sensitivity was previously reported inaccurately as $1.3 \%$ (18) because of misreporting of the source activity by the manufacturer. Figure 4 shows the mouse NECR as a function of source activity, along with corresponding rates of trues, scatters, and randoms. The NECR at $3.7 \mathrm{MBq}$ was $11.1 \mathrm{kcps}$, and a peak NECR of $20.8 \mathrm{kcps}$ was reached at $14.5 \mathrm{MBq}$. The scatter fraction at $3.2 \mathrm{MBq}$ was $15.4 \%$.

Zoom-1 FBP-3DRP and PSF-MLEM (30 iterations) reconstructions of the 3 sections of the NEMA image-quality phantom are shown in Supplemental Figure 1 (supplemental materials are available at http://jnm.snmjournals.org). All 5 rods were visible in the hot-rod section, and the uniformity section appeared uniform. The images, which were not quantitative because of lack of deadtime correction, were rescaled to have approximately the same value in regions where bed positions overlapped. Uniformity, recovery coefficients, and spillover ratios resulting from both FBP3DRP and PSF-MLEM reconstructions are shown in Table 4. The recovery coefficients were generally higher when PSF-MLEM was used.

Figure 5 shows zoom-2 reconstructions of the microresolution phantom made with FBP-3DRP (left) and 99 iterations of PSFMLEM (right). The FBP-3DRP reconstruction resolved rod sizes as small as $0.9 \mathrm{~mm}$, whereas the PSF-MLEM reconstruction resolved even the smallest rods, which were $0.7 \mathrm{~mm}$ wide. At a resolution so high that resolvable feature sizes are comparable to the average positron range $\left(0.85 \mathrm{~mm}\right.$ for $\left.{ }^{18} \mathrm{~F}(34)\right)$, further improvements

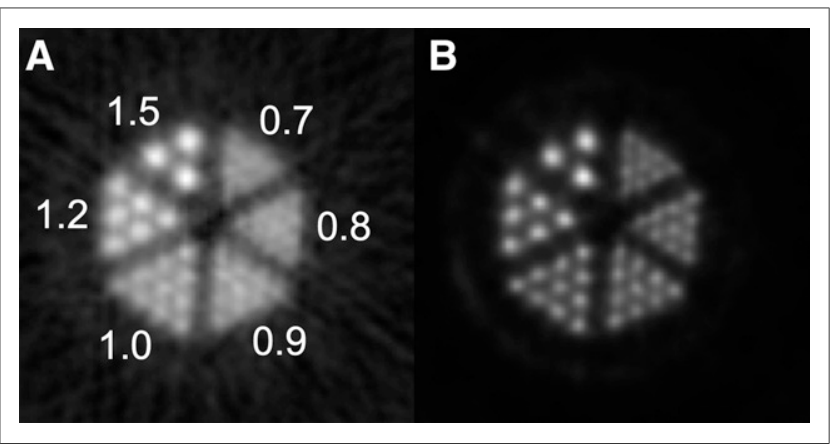

FIGURE 5. Reconstructions of microresolution phantom made using FBP-3DRP (A) and PSF-MLEM (B). Both images are cropped to $32 \times$ $32 \mathrm{~mm}$ area. Insert diameters are indicated in millimeters. to the algorithm would likely need to account for positron range in the system matrix (35).

Zoom-1 PSF-MLEM reconstructions of the mouse and rat brains (respectively, using 21 and 25 iterations) are shown in Figure 6, along with fused MR images. Corresponding FBP-3DRP reconstructions are shown in Supplemental Figure 2. PSF-MLEM produced rodent images that were much more capable of resolving structures within the brain, showing clear separation between the cortex and the basal ganglia. As expected, regions in the MR images that were clearly identifiable as cortex corresponded to high ${ }^{18} \mathrm{~F}$-FDG uptake in the PET images.

\section{DISCUSSION}

An MR-compatible small-animal PET insert was characterized in terms of spatial resolution, sensitivity, and NECR. Spatial resolution in the center of the FOV outperformed that of the commonly used Siemens microPET Focus 120, Focus 220, and Inveon preclinical PET scanners, for which the respective radial resolutions at 5-mm offsets are 1.92, 1.75, and $1.63 \mathrm{~mm} \mathrm{(36).} \mathrm{At} \mathrm{a}$ 15-mm offset, these systems compared closely to ours in terms of radial resolution $(1.99,1.82$, and $2.03 \mathrm{~mm})$. The spatial resolution of the MR-compatible preclinical PET insert reported by Ko et al. (12) is nominally the same as ours in the center of the FOV but degrades with radial offset faster than ours because of lack of depth-of-interaction measurement (volumetric resolution of 1.93 and $7.27 \mu \mathrm{L}$ at 0 - and $14-\mathrm{mm}$ radial offsets, respectively). The Hyperion-II $^{\mathrm{D}}$ (a digital-SiPM-based MR-compatible PET insert) was shown to resolve a rod size as small as only $0.8 \mathrm{~mm}$ using an MLEM reconstruction algorithm (14)—slightly below the performance of our PET system.

NECR and peak sensitivity were notably poorer than in other preclinical PET systems; however, this was largely due to the low geometric factor following from the limited axial FOV. The peak sensitivity of the microPET Focus 120, Focus 220, and Inveon are, respectively, $3.42 \%, 2.28 \%$, and $6.72 \%$ (36), compared with $2.2 \%$ for our system when using a similar energy window. At $3.7 \mathrm{MBq}$, these systems have a mouse NECR of 66.5, 47.3, and $129.0 \mathrm{kcps}$, respectively, all exceeding the $11.1 \mathrm{kcps}$ that we measured. The Hyperion-II ${ }^{\mathrm{D}}$ has a peak sensitivity of $2.6 \%$ using a lower energy limit of $250 \mathrm{keV}$ (37). The MR-compatible PET insert by Ko et al. has a reported peak sensitivity of $3.36 \%$ (38) and a mouse NECR of $23.4 \mathrm{kcps}$ at $3.7 \mathrm{MBq}$ (12) while using a lower energy limit of $250 \mathrm{keV}$. This sensitivity figure is comparable to that of our system. Analysis of singles data suggests that our system is characterized by paralyzable block-level dead time in excess of approximately $1.3 \mu \mathrm{s}$. Such a large dead time would compound with our low geometric 


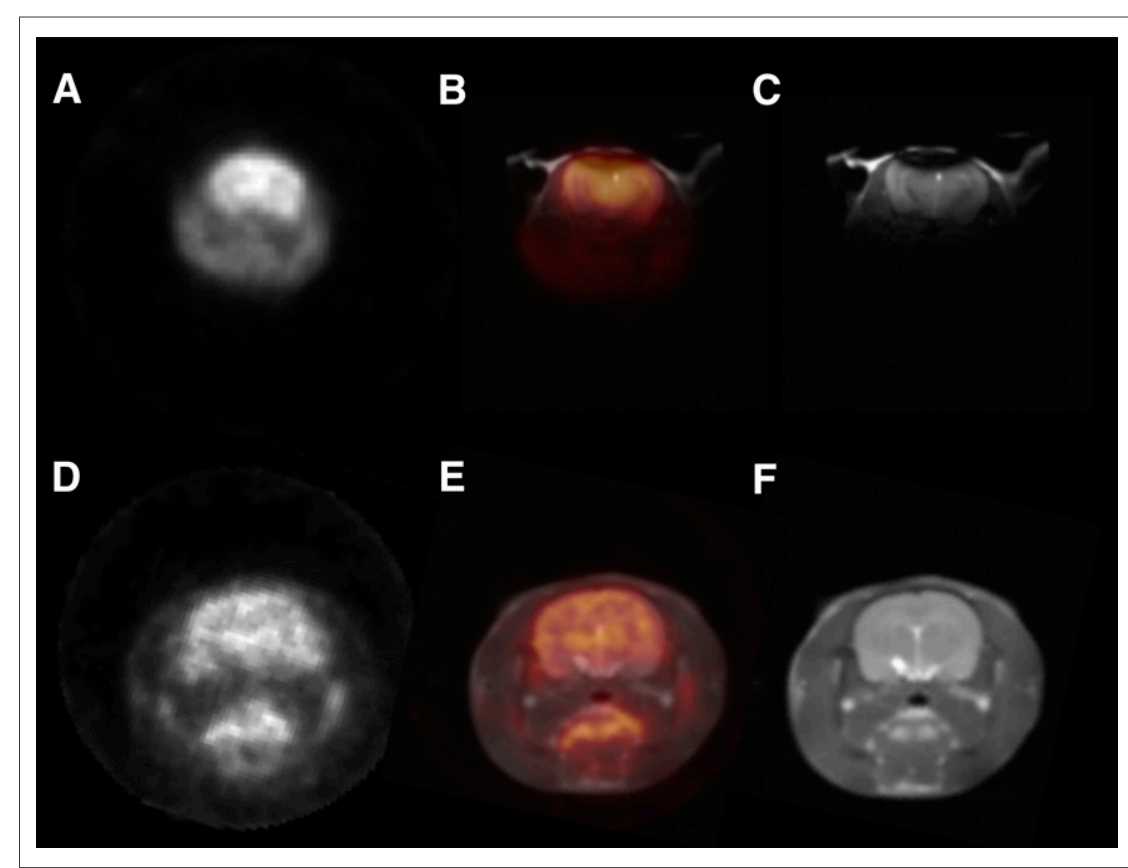

FIGURE 6. (A-C) ${ }^{18}$ F-FDG PET image of mouse brain reconstructed with PSF-MLEM (A), PET image fused with MR image (B), and unfused MR image (C). (D-F) Corresponding images for rat brain. All images are cropped to $42 \times 42 \mathrm{~mm}$ area. used for the rat imaging was supplied by Cubresa Inc. at no cost. No other potential conflict of interest relevant to this article was reported.

\section{ACKNOWLEDGMENTS}

We thank Drs. Melanie Martin and Richard Buist from the University of Manitoba In-Vivo Experimental Animal Magnetic Resonance Microscopy Centre for assisting with PET/MR imaging, Dr. Michael Jackson of the University of Manitoba Small Animal and Materials Imaging Core Facility for assisting with animal handling, Drs. Dali Zhang and Ji Hyun Ko from the Neuroscience Research Program at the University of Manitoba for assisting with rat imaging, Dr. Benedict Albensi from the Department of Pharmacology and Therapeutics at the University of Manitoba for supplying the mice, and Bob Miller of the Medical Devices Group at CancerCare Manitoba for assisting with PET insert gantry design and fabrication. sensitivity to explain our low NECR performance. Future versions of our PET insert design will have an axial FOV between 2 and 3 times longer than the prototype and reduced dead time, resulting in large gains in sensitivity and NECR.

PET images acquired simultaneously with MRI and vice versa showed no signs of interference. In a previous study of the MR compatibility of our PET insert (17), no significant effect on the PET system due to the MRI was observed, and the PET system introduced only minor degradations in $\mathrm{B}_{0}$ homogeneity (0.16$0.26 \mathrm{ppm}$ ) and no significant drop in image signal-to-noise-ratio during multislice, multi-echo; rapid-imaging-with-refocusedechoes; and fast-low-angle-shot pulse sequences. However, a $9 \%$ drop in signal-to-noise ratio was observed for echo-planar imaging sequences.

\section{CONCLUSION}

This MR-compatible PET insert can obtain high-resolution PET images while functioning inside a 7-T MRI scanner with very little intermodality interference. PET image quality is especially good when the custom-made PSF-MLEM reconstruction software is used. NECR and sensitivity are low compared with most PET systems because of the short axial length of the scanner and high dead time of the current prototype.

\section{DISCLOSURE}

This work was funded by Mitacs Accelerate Cluster Grant IT04494 to Vesna Sossi and Andrew L. Goertzen partnered with Cubresa Inc. (Winnipeg, Canada); Natural Sciences and Engineering Research Council of Canada Discovery Grants to Christopher J. Thompson (36672), Andrew L. Goertzen (341628), and Vesna Sossi (240670); and the University of Manitoba Amalgamated Research Fund. The radiofrequency coil

\section{REFERENCES}

1. Hutchins GD, Miller MA, Soon VC, Receveur T. Small animal PET imaging. ILAR J. 2008;49:54-65.

2. Shao Y, Cherry SR, Farahani K, et al. Simultaneous PET and MR imaging. Phys Med Biol. 1997;42:1965-1970.

3. Catana C, Wu Y, Judenhofer MS, et al. Simultaneous acquisition of multislice PET and MR images: initial results with a MR-compatible PET scanner. J Nucl Med. 2006;47:1968-1976.

4. Farahani K, Slates R, Shao Y, et al. Contemporaneous positron emission tomography and MR imaging at 1.5 T. J Magn Reson Imaging. 1999;9:497-500.

5. Raylman RR, Majewski S, Lemieux SK, et al. Simultaneous MRI and PET imaging of a rat brain. Phys Med Biol. 2006;51:6371-6379.

6. Yamamoto S, Imaizumi M, Kanai Y, et al. Design and performance from an integrated PET/MRI system for small animals. Ann Nucl Med. 2010;24: 89-98.

7. Maramraju SH, Smith SD, Junnarkar SS, et al. Small animal simultaneous PET/ MRI: initial experiences in a 9.4 T microMRI. Phys Med Biol. 2011;56: 2459-2480.

8. Judenhofer MS, Catana C, Swann BK, et al. PET/MR images acquired with a compact MR-compatible PET detector in a 7-T magnet. Radiology. 2007;244: 807-814.

9. Pichler BJ, Judenhofer MS, Catana C, et al. Performance test of an LSO-APD detector in a 7-T MRI scanner for simultaneous PET/MRI. J Nucl Med. 2006;47:639-647.

10. Delso G, Furst S, Jakoby B, et al. Performance measurements of the Siemens mMR integrated whole-body PET/MR scanner. J Nucl Med. 2011;52:19141922.

11. Roncali E, Cherry SR. Application of silicon photomultipliers to positron emission tomography. Ann Biomed Eng. 2011;39:1358-1377.

12. Ko GB, Kim KY, Yoon HS, et al. Evaluation of a silicon photomultiplier PET insert for simultaneous PET and MR imaging. Med Phys. 2016;43:72-83.

13. Yamamoto S, Watabe T, Watabe H, et al. Simultaneous imaging using Si-PMbased PET and MRI for development of an integrated PET/MRI system. Phys Med Biol. 2012;57:N1-N13.

14. Schug D, Lerche C, Weissler B, et al. Initial PET performance evaluation of a preclinical insert for PET/MRI with digital SiPM technology. Phys Med Biol. 2016;61:2851-2878.

15. Heering A, Rohlf J, Freeman J, et al. Performance of silicon photomultipliers with the CMS HCAL front-end electronics. Nucl Instrum Meth A. 2007;576:341-349. 
16. Spanoudaki VC, Mann AB, Otte AN, et al. Use of single photon counting detector arrays in combined PET/MR: characterization of LYSO-SiPM detector modules and comparison with a LSO-APD detector [abstract]. J Instrum. 2007;2:P12002.

17. Thiessen JD, Shams E, Stortz G, et al. MR-compatibility of a high-resolution small animal PET insert operating inside a 7 T MRI. Phys Med Biol. 2016;61:7934-7956.

18. Goertzen AL, Stortz G, Thiessen JD, et al. First results from a high-resolution small animal SiPM PET insert for PET/MRI imaging. IEEE Trans Nucl Sci. 2016;63:2424-2433.

19. Zhang X, Stortz G, Sossi V, et al. Development and evaluation of a LOR-based image reconstruction with $3 \mathrm{D}$ system response modeling for a PET insert with dual-layer offset crystal design. Phys Med Biol. 2013;58:8379-8399.

20. Thompson CJ, Stortz G, Goertzen AL, et al. Comparison of single and dual layer detector blocks for pre-clinical MRI-PET. Med Phys. 2012;39:4641-4642.

21. Thompson CJ, Goertzen AL, Berg E, et al. Evaluation of high density pixellated crystal blocks with SiPM readout as candidates for PET/MR detectors in a small animal PET insert. IEEE Trans Nucl Sci. 2012;59:1791-1797.

22. Goertzen AL, Zhang X, McClarty MM, et al. Design and performance of a resistor multiplexing readout circuit for a SiPM detector. IEEE Trans Nucl Sci. 2013;60:1541-1549.

23. Liu CY, Goertzen AL. Multiplexing approaches for a $12 \times 4$ array of silicon photomultipliers. IEEE Trans Nucl Sci. 2014;61:35-43.

24. Goertzen AL, Thiessen JD, Zhang X, et al. Application of HDMI® cables as an MRI compatible single cable solution for readout and power supply of SiPM based PET detectors. IEEE Nucl Sci Conf R. 2012:3184-3188.

25. Abu-Nimeh FT, Ito J, Moses WW, et al. Architecture and implementation of OpenPET firmware and embedded software. IEEE Trans Nucl Sci. 2016;63:620-629.

26. Moses WW, Buckley S, Vu C, et al. OpenPET: a flexible electronics system for radiotracer imaging. IEEE Trans Nucl Sci. 2009;2009:3491-3495.
27. Schellenberg G, Stortz G, Goertzen AL. An algorithm for automatic crystal identification in pixelated scintillation detectors using thin plate splines and Gaussian mixture models. Phys Med Biol. 2016;61:N90-N101.

28. Stortz G. Development of a Small Animal MR Compatible PET Insert [thesis]. Vancouver, Canada: University of British Columbia; 2016.

29. Fahey FH. Data acquisition in PET imaging. J Nucl Med Technol. 2002;30: 39-49.

30. Thielemans K, Tsoumpas C, Mustafovic S, et al. STIR: software for tomographic image reconstruction release 2. Phys Med Biol. 2012;57:867-883.

31. Bai B, Li Q, Holdsworth $\mathrm{CH}$, et al. Model-based normalization for iterative 3D PET image reconstruction. Phys Med Biol. 2002;47:2773-2784.

32. National Electrical Manufacturers Association. NEMA Standard Publication NU 4-2008: Performance Measurements of Small Animal Positron Emission Tomographs. Rosslyn, VA: National Electrical Manufacturers Association; 2008.

33. Goertzen AL, Buist R, Herrera SL, et al. A custom RF coil for high resolution PET/MR imaging at 7T with a PET insert. Presented at: PSMR2016-5th Conference on "PET/MR and SPECT/MR"; Cologne, Germany; 2016. In press.

34. Tai YC, Laforest R. Instrumentation aspects of PET imaging. Annu Rev Biomed Eng. 2005;7:285-288.

35. Rahmim A, Qi J, Sossi V. Resolution modeling in PET imaging: theory, practice, benefits, and pitfalls. Med Phys. 2013;40:064301.

36. Goertzen AL, Bao Q, Bergeron M, et al. NEMA NU 4-2008 comparison of preclinical PET imaging systems. J Nucl Med. 2012;53:1300-1309.

37. Weissler B, Gebhardt P, Dueppenbecker PM, et al. A digital preclinical PET/ MRI insert and initial results. IEEE Trans Med Imaging. 2015;34:22582270 .

38. Ko GB, Yoon HS, Kim KY, et al. Simultaneous multi-parametric PET/MRI with silicon photomultiplier PET and ultra-high field MRI for small animal imaging. J Nucl Med. 2016;57:1309-1315. 